\title{
Folding of Proteins in Go Models with Angular Interactions
}

\author{
Marek Cieplak ${ }^{\mathrm{a}}$ and Trinh Xuan Hoang b,c \\ ${ }^{a}$ Institute of Physics, Polish Academy of Sciences, Al. Lotników 32/46, 02-668 \\ Warsaw, Poland \\ ${ }^{\mathrm{b}}$ The Abdus Salam International Centre for Theoretical Physics, Strada Costiera \\ 11, 34100 Trieste, Italy \\ ${ }^{\mathrm{c}}$ Institute of Physics, NCST, P.O. Box 429, Bo Ho, Hanoi 10000, Vietnam
}

\begin{abstract}
Molecular dynamics studies of Go models of proteins with the 10-12 contact potential and the bond and dihedral angle terms indicate statistical similarities to other Go models, e.g. with the Lennard-Jones contact potentials. The folding times depend on the protein size as power laws with the exponents depending on the native structural classes. There is no dependence of the folding times on the relative contact order even though the folding scenarios are governed mostly by the contact order.
\end{abstract}

Key words: Protein folding, molecular dynamics, Go model

PACS: 87.10.+e, 87.15.-v

\section{Introduction}

There is a general conviction that protein folding is governed by the geometry of the protein $[1,2,3,4]$ and especially by the geometry of its native state $[5,6,7]$. On the other hand, it is a matter of a debate $[6,7,8,9,10,11,12,13]$ when it comes to deciding on what does the folding time specifically depend on, what are the proper measures of the protein's geometry that are relevant for the kinetics, and is there a dependence on the system size. In particular, a compilation of folding times at room temperature [7] has suggested no dependence on the number, $N$, of aminoacids and existence of a correlation with the so called relative contact order, $C O$. This parameter is a normalized average length along the sequence between pairs of amino acids that interact, or make a contact. It should be pointed out, however, that the evidence for the dependence on $\mathrm{CO}$ in the $\beta$-proteins is rather weak [13]. The $\beta$-proteins provide a 
crucial test case because their $\mathrm{CO}$ is dominated by long range contacts. Furthermore, a definition of a characteristic folding time for a protein need not necessarily be related to a room temperature measurement. Instead, it may require a determination of optimal kinetic conditions for folding $[10,13,14]$ and, in particular, of the optimal kinetic temperature, $T_{\min }$.

In this context, it is interesting to determine what are the theoretical predictions of the coarse-grained Go-like $[15,16]$ models since their construction depends primarily on the geometry of the native state and thus these models should be particularly attuned to the features of geometry. In ref. [13], we have studied the scaling properties of the folding times of 51 proteins in two versions of the Go model. In the current paper, we report similar results on the third model, henceforth called model C, which has been used by Clementi, Nymeyer, and Onuchic [17] for several proteins. It has also been used by Koga and Takada [10] to study folding times of many proteins at temperatures corresponding to the maximal specific heat and to propose a correlation with $\mathrm{CO} \cdot N^{0.6}$, as we discuss further in reference [13].

Model $\mathrm{C}$, in addition to the pairwise distance-dependent interactions of the 10-12 form and harmonic tethering forces between subsequent amino acids, incorporates three and four body terms in the potential which correspond to angular dependencies. This model has been shown to posses an explicit two-state behavior since its free energy, at least for some proteins, has a twominima form when plotted against the fraction, $Q$, of the established native contacts. Thus in the model of Clementi et al., $Q$ is believed to be the reaction coordinate for folding whereas in the other models studied in ref. [13] the explicit reaction coordinate is not known. We find that all three models show nearly identical behavior even though individual values of the parameters may display sensitivity to the choice of the model. In particular, there is a division into (at least) three kinetic universality classes which agree with the three structural classes: $\alpha-, \beta$-, and $\alpha-\beta$ proteins, where the division is based on what are the kinds of secondary structures that are present in the native state. In each class, there is a separate growing trend of the folding time with $N$. This trend appears to be better described by a power law than by an exponential function.

Another result for model $\mathrm{C}$ is that there is still no correlation with $\mathrm{CO}$. It should be noted that the folding scenarios can be described in terms of graphs that show times, $t_{c}$, needed to establish contacts plotted againts the contact lengths. In all three models, these graphs are dominated by a monotonically increasing function of $j-i$, where $i$ and $j$ are the locations of the amino acids involved. A strictly monotonic dependence can be characterized by a single 
parameter, like CO, but the deviations are outside of the scope of such a description. It is the usually present deviations from the monotonic trends that should be responsible for the lack of correlation with CO. An alternative point of view though has been recently proposed by Plaxco et al. [18] and it is that simple Go models lack cooperativity. They suggest that this cooperativity can be introduced by making contact energies depend on conformation through $Q$ and studies of $N=27$ lattice models suggest a correlation with CO.

\section{Go models}

The general basic prescription for the construction of a Go model of a protein is to use a Hamiltonian that incorporates the chain-like connectivity and such that the ground state of the model agrees with the experimentally determined native conformation. Clearly, there are many ways of accomplishing this but they are expected to be physically similar. Our approach is outlined in references $[19,20]$ with the updated details given in [13]. Briefly, the amino acids are represented by point particles of mass $m$ located at the positions of the $\mathrm{C}^{\alpha}$ atoms. They are tethered by a strong harmonic potential with a minimum at the peptide bond length. The native structure of a protein is taken from the PDB [21] data bank and the interactions between the amino acids are grouped into native and non-native. The distinction is based on taking the fully atomic representation of the amino acids in the native state and then checking for overlaps assuming the van der Waals radii of the atoms. The procedure [22,23] involves multiplication of the radii by 1.24 to take softness of the potential into account. The amino acids ( $i$ and $j$ ) that are found to overlap in this sense are considered to be forming contacts and the corresponding contact range is from about 4.4 to $12.8 \AA$. These pairs are endowed with a pairwise attractive potential, $V_{i j}$, between the $C^{\alpha}$ atoms such that its minimum agrees with the experimentally determined distance in the native state. The non-native contacts are purely repulsive and corespond to a core of radius $\sigma=5 \AA$.

The choice of the attractive native potential and a selection of additional terms in the Hamiltonian is what makes a distinction between various versions of the model. In model $\mathrm{A}$

$$
V_{i j}=4 \epsilon\left[\left(\frac{\sigma_{i j}}{r_{i j}}\right)^{12}-\left(\frac{\sigma_{i j}}{r_{i j}}\right)^{6}\right]
$$


whereas in models $\mathrm{B}$ and $\mathrm{C}$

$$
V_{i j}=\epsilon\left[\left(5 \frac{r_{i j}^{(n)}}{r_{i j}}\right)^{12}-6\left(\frac{r_{i j}^{(n)}}{r_{i j}}\right)^{10}\right],
$$

where $r_{i j}^{(n)}$ coincides with the native distance. This potential is believed to correspond to the hydrogen bonds better than the Lennard-Jones one. $\epsilon$ is the uniform scale of the energy. In models $\mathrm{A}$ and $\mathrm{B}$ the additional terms correspond to four body forces that favor the native sense of chirality [13]. Model C is our primary focus in this paper and instead of the chirality potential it is equipped with the angular terms:

$$
\begin{aligned}
V^{B A} & =\sum_{i=1}^{N-2} K_{\theta}\left(\theta_{i}-\theta_{0 i}\right)^{2} \\
V^{D A} & =\sum_{i=1}^{N-3}\left[K_{\phi}^{1}\left(1+\cos \left(\phi_{i}-\phi_{0 i}\right)+K_{\phi}^{3}\left(1+\cos 3\left(\phi_{i}-\phi_{0 i}\right)\right)\right]\right.
\end{aligned}
$$

where $\theta_{i}$ and $\phi_{i}$ represent the bond and dihedral angles respectively and the subscript 0 indicates the native values. The bond angle is determined by three subsequent residues and the dihedral angle by four subsequent residues (by forming vector products of two subsequent residue-to-residue position vectors). Following ref. [17], we take $20 \epsilon, \epsilon$, and $0.5 \epsilon$ for $K_{\theta}, K_{\phi}^{1}$, and $K_{\phi}^{3}$ respectively and the native contacts with $|i-j|<4$ are discarded.

The ground state of the model corresponds to the native state at room temperature. The thermal fluctuations away form this state are accounted for by introducing the Langevin noise [24] with the damping constant $\gamma$ of $2 \mathrm{~m} / \tau$, where $\tau$ is $\sqrt{m \sigma^{2} / \epsilon}$. This corresponds to the situation in which the inertial effects are negligible [13] but a more realistic account of the water environment requires $\gamma$ to be about 25 times larger [25]. Thus the folding times obtained for $\gamma=2 m / \tau$ need to be multiplied by 25 since there is a linear dependence on $\gamma[19,20]$.

\section{$3 \quad$ Results}

The list of the proteins studied is exactly the same as in ref. [13]. 21, 14, and 16 are of the $\alpha-\beta, \alpha$, and $\beta$ kind respectively. The temperature dependence of the folding time was determined as a median time corresponding to at least 
101 folding processes. The median folding time, as determined at $T_{\min }$, is denoted by $t_{\text {fold }}$. The results for model $\mathrm{C}$ on the log-log and log-linear scales are shown in Figures 1 and 2 respectively. Each panel corresponds to a different structural class. The $\alpha$ - proteins exhibit the slowest growth with $N$. Compared to models A and B [13], the folding times for individual proteins may be quite distinct, but the general location of the data points and the trends are the same. Thus, statistically, all of the three models are equivalent and the growing trends with $N$ are the same. One way to illustrate this is shown in Figure 2 in which the folding times obtained in model $\mathrm{C}$ are plotted against those in model A. The data points are clustered around the diagonal direction and the correlation level is $85 \%$.

The power law fits (Figure 1) correspond to the exponents of 2.5, 1.7 and 3.2 for $\alpha-\beta, \alpha$, and $\beta$ respectively. The exponential law fits (Figure 3) yield correlation lengths correspondingly of 32,42 and 24. All of these values are consistent with what was obtained for models $\mathrm{A}$ and $\mathrm{B}$ and the fits to the exponential laws are worse (the overall correlation level for the power laws in model $\mathrm{C}$ is $81 \%$ but $74 \%$ for the exponential fits) However, in model $\mathrm{C}$, the scatter of the data points around the trends is somewhat larger than in the other two models (the corresponding sets of numbers are $86 \%, 82 \%$ and $89 \%$, $87 \%$ for models A and B respectively).

Figure 4 shows the behavior of characteristic temperatures, $T_{f}$ and $T_{\min }$, for the 51 proteins. The former is the folding temperature. It is determined by the condition that the probability of staying in the native basin is near $\frac{1}{2}$. Compared to models A and B, the values of the two temperatures are, roughly, twice as high but they stay comparable to each other, indicating overall good folding properties (in models of random sequences of amino acids, $T_{f}$ is substantially lower than $\left.T_{\text {min }}\right)$. Similar to models $\mathrm{A}$ and $\mathrm{B}$, there are no growing trends with $N$ in $T_{f}$. In model A, there appears to be a growing trend in $T_{\min }$ for the $\alpha$ and $\alpha-\beta$ proteins [13]. In model C, on the other hand (Figure 4), there is either no net dependence on $N$ or a weak growing trend in $T_{\min }$ for each structural class.

It should be noted that the twice as big characteristic temperatures found in model $\mathrm{C}$, when compared to model $\mathrm{A}$, also affect a scale of a typical dependence of the folding time, $t_{\text {fold }}$, on $T$. This is illustrated for the protein 2ci2 in Figure 5. In model $\mathrm{C}$, the dependence is much broader than in model $\mathrm{A}$ and this is the usual situation. There are proteins, however, like 1aho, in which the opposite takes place and then $t_{\text {fold }}$ at $T_{\text {min }}$ in model $\mathrm{A}$ is shorter (by a factor of 2.5 in this case) than in C. There are also proteins, like crambin, for which the widths of the U-shaped curves are about the same and so are the folding 
times at $T_{\min }$.

The dependence on $\mathrm{CO}$ is also very much like in models $\mathrm{A}$ and B. Figure 6 shows that neither $t_{\text {fold }}$ nor the characteristic temperatures exhibit any clear trend with $\mathrm{CO}$, except maybe for a very weak growth of $T_{f}$.

The folding scenarios, however, do depend on the contact order. This is illustrated in Figure 7 for the case of the proteins with the PDB code of 2ci2 and for all of the three models. Independent of the model, there is a basic monotonic growth of the average time needed to establish a contact for the first time. The average is obtained based on 400 folding trajectories at $T_{\text {min }}$. Most events are governed by this monotonic trend but there is a lower side branch which introduces double valuedness in the dependence on $|j-i|$. Thus the contact order cannot fully describe the folding process which explains no dependence on CO. It is remarkable that the folding scenarios are so insensitive to the detailed version of the Go model.

Model C studied here is nearly the same as the one considered by Clementi et al. [17] but there are two differences. The first one is that the contact maps are not exactly the same because of the different procedures used to determine the contacts. The second is a different molecular dynamics scheme. Clementi et al. use a leapfrog algorithm and control the temperature by velocity rescaling. Ours is the fifth order predictor-corrector scheme with the Langevin noise as a thermostat. As expected, our results for the equilibrium quantities are very close. This is illustrated in Figure 8 which shows the free energy as a function of $Q$ and specific heat as a function of $T$ for the protein 2ci2, both obtained by the histogram method [26,27]. The two-state behavior and values of the quantities are in agreement with Clementi et al. The kinetic properties, however, need not agree due to a different account of the effective viscosity of the environment.

In summary, the choice of a version of the Go model, within the same molecular dynamics scheme, is not very relevant in statistical studies of model proteins even though it may affect properties of individual systems. Each Go model suggests same power law dependence on $N$ of the folding time and no dependence on a single average contact order. It would be interesting to study whether the cooperativity effects, as discussed by Plaxco et al. [18] can indeed generate demonstrable trends as a function of CO.

This work was funded by KBN. 


\section{References}

[1] See, e.g., C. Micheletti, J. R. Banavar, A. Maritan, and F. Seno, Phys. Rev. Lett. 82 3372-3375 (1999).

[2] A. Maritan, C. Micheletti, A. Trovato, and J. R. Banavar, Nature 406287 (2000).

[3] J. R. Banavar and A. Maritan, Rev. Mod. Phys. 75 23-34 (2003).

[4] R. Du, V. S. Pande, A. Y. Grosberg, T. Tanaka, and E. I. Shakhnovich, J. Chem. Phys. 111 10375-10380 (1999).

[5] R. Unger and J. Moult, J. Mol. Biol. 259 988-994 (1996).

[6] K. W. Plaxco, K. T. Simons, and D. Baker, J. Mol. Biol. 277 985-994 (1998).

[7] K. W. Plaxco, K. T. Simons, I. Ruczinski, D. Baker, Biochemistry 39 1117711183 (2000).

[8] D. Thirumalai, J. Physique I 5 1457-1467 (1995).

[9] A. M. Gutin,V. I. Abkevich, and E. I. Shakhnovich, Phys. Rev. Lett. 77 54335436 (1996).

[10] N. Koga and S. Takada, J. Mol. Biol. 313 171-180 (2001).

[11] V. P. Zhdanov, Europhys Lett. 42 577-581 (1998).

[12] M. Cieplak, T. X. Hoang, and M. S. Li, Phys. Rev. Lett. 83 1684-1687 (1999).

[13] M. Cieplak and T. X. Hoang, Biophysical Journal 84 475-488 (2003).

[14] M. Cieplak and T. X. Hoang, Proteins, 44 20-25 (2001).

[15] H. Abe, N. Go, Biopolymers 20, 1013-1031 (1981).

[16] S. Takada, Proc. Natl. Acad. Sci. USA 96, 11698-11700 (1999).

[17] C. Clementi, H. Nymeyer, and J. N. Onuchic. J. Mol. Biol. 298 937-953 (2000).

[18] A. I. Jewett, V. S. Pande, and K. W. Plaxco, J. Mol. Biol. 326 247-253 (2003).

[19] T. X. Hoang and M. Cieplak, J. Chem. Phys. 112, 6851-6862 (2000).

[20] T. X. Hoang and M. Cieplak, J. Chem. Phys. 113, 8319-8328 (2001).

[21] F. C. Bernstein, T. F. Koetzle, G. J. B. Williams, E. F. Meyer Jr., M. D. Brice, J. R. Rodgers, O. Kennard, T. Shimanouchi, and M. Tasumi, J. Mol. Biol. 112, 535-542 (1977).

[22] J. Tsai, R. Taylor, C. Chothia, and M. Gerstein, J. Mol. Biol. 290, 253-266 (1999). 
[23] G. Settanni, T. X. Hoang, C. Micheletti, and A. Maritan, Biophys. J. 83, 35333541 (2002).

[24] G. S. Grest, K. Kremer, Phys. Rev. A 33, 3628-3631 (1986).

[25] T. Veitshans, D. Klimov, and D. Thirumalai, Folding Des. 2, 1-22 (1997).

[26] A. M. Ferrenberg, and R. H. Swendsen, Phys. Rev. Lett. 63 1195-1198 (1989).

[27] S. Kumar, D. Bouzida, R. H. Swendsen, P. A. Kollman, J. M. Rosenberg, J. Comp. Chem. 13 1011-1021 (1992). 


\section{FIGURE CAPTIONS}

Fig. 1. The scaling of $t_{\text {fold }}$ with $N$ for the 51 proteins in model C. The data are split into the structural classes as indicated. The lines correspond to th epower law exponent displayed in the right corner of each panel. The error bars on the exponent are of order \pm 0.2 . The folding times are calculated at $T_{m i n}$. The correlation levels of the points shown are $78 \%, 98 \%$, and $87 \%$ for the top, middle, and bottom panels respectively.

Fig. 2. The log-log plot of the folding times at $T_{\min }$ for the 51 proteins as obtained in model $\mathrm{C}$ versus those obtained in model $\mathrm{A}$.

Fig. 3. The data of Figure 1 redisplyed on the log-linear plane. The dashed line indicate fits to the exponential law $t_{\text {fold }} \sim \exp (b / \xi)$ with the values of $\xi$ shown in the right corner of each panel. The correlation levels are $68 \%$, $97 \%$, and $81 \%$ respectively.

Fig. 4. The values of $T_{\min }$ and $T_{f}$ shown versus $N$ for model $\mathrm{C}$ for the three structural classes. The solid lines indicate the average values. The dotted lines show the average values of $T_{g 2}$ - the temperature at which the median folding time is twice as long as at $T_{\min }$ and $T_{g 2}$ is on the lower temperature side of $T_{\min }$.

Fig. 5. The folding time, defined as the first passage time, for the protein 2 ci2 as a function of $T$ in the two models. The solid guiding line and filled circles are for model $\mathrm{C}$ and the dotted line with the open square symbols are for model A. The arrows indicate the values of $T_{f}$. The values of $T_{\min }$ are 0.25 and $0.48 \epsilon / k_{B}$ for models $\mathrm{A}$ and $\mathrm{C}$ respectively.

Fig. 6. The dependence of $t_{\text {fold }}, T_{m i n}$, and $T_{f}$ on the relative contact order parameter for model $\mathrm{C}$. The data symbols indicate the structural classes and are identical to thise in Figs. 1,2, and 3.

Fig. 7. The average times to form contacts of a given length $|j-i|$ for the first time in the three models. The error bars are of the order of the size of the symbols.

Fig. 8. The thermodynamic parameter for $2 \mathrm{ci} 2$ in model C. The top panel shows the specific heat (the maximum is located at a temperature which is nearly twice as high as $T_{f}$ ). The bottom panel shows the free energy as a function of the fraction of established native contacts at the temperature corresponding to the maximum in the specific heat. 


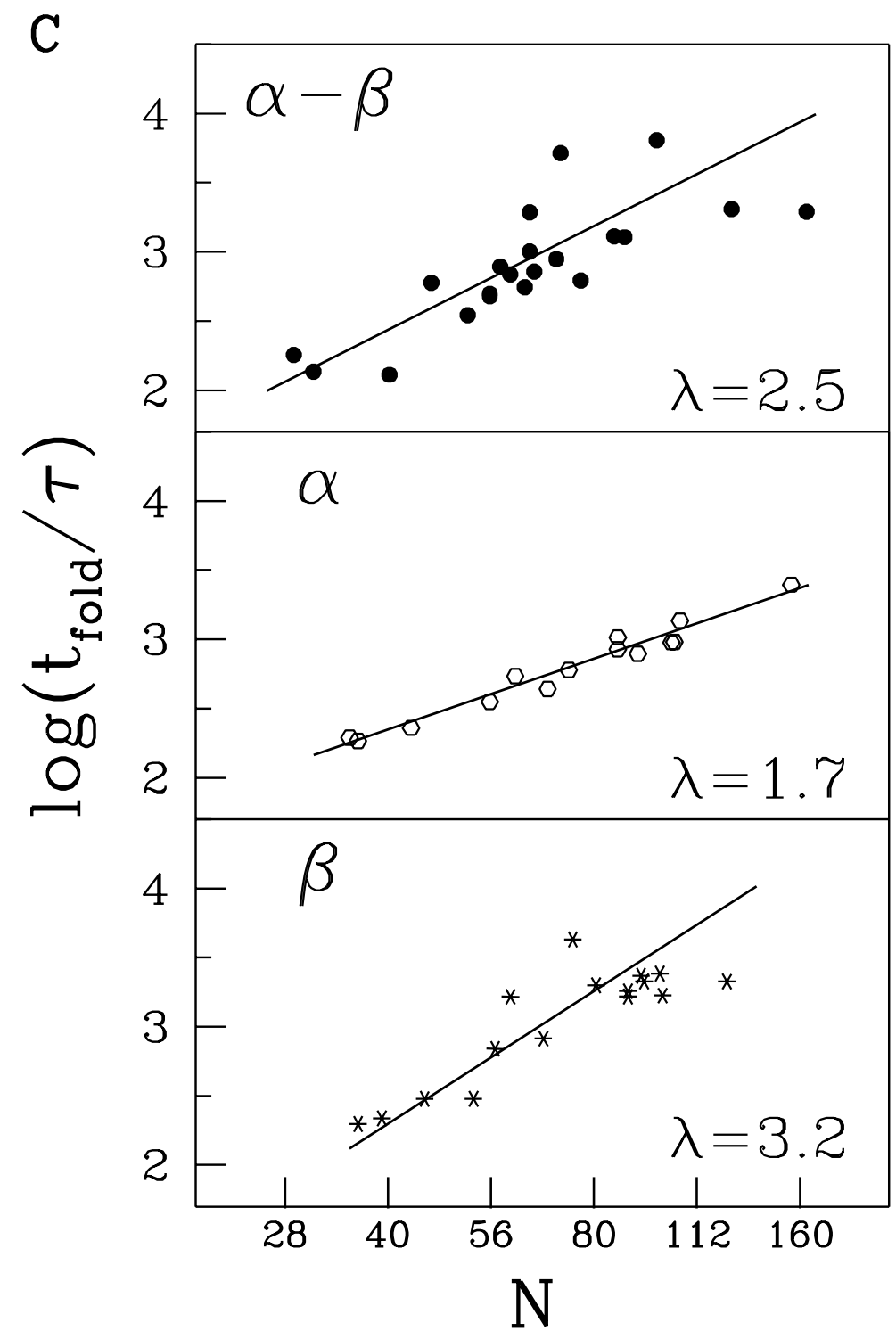

Fig. 1. 


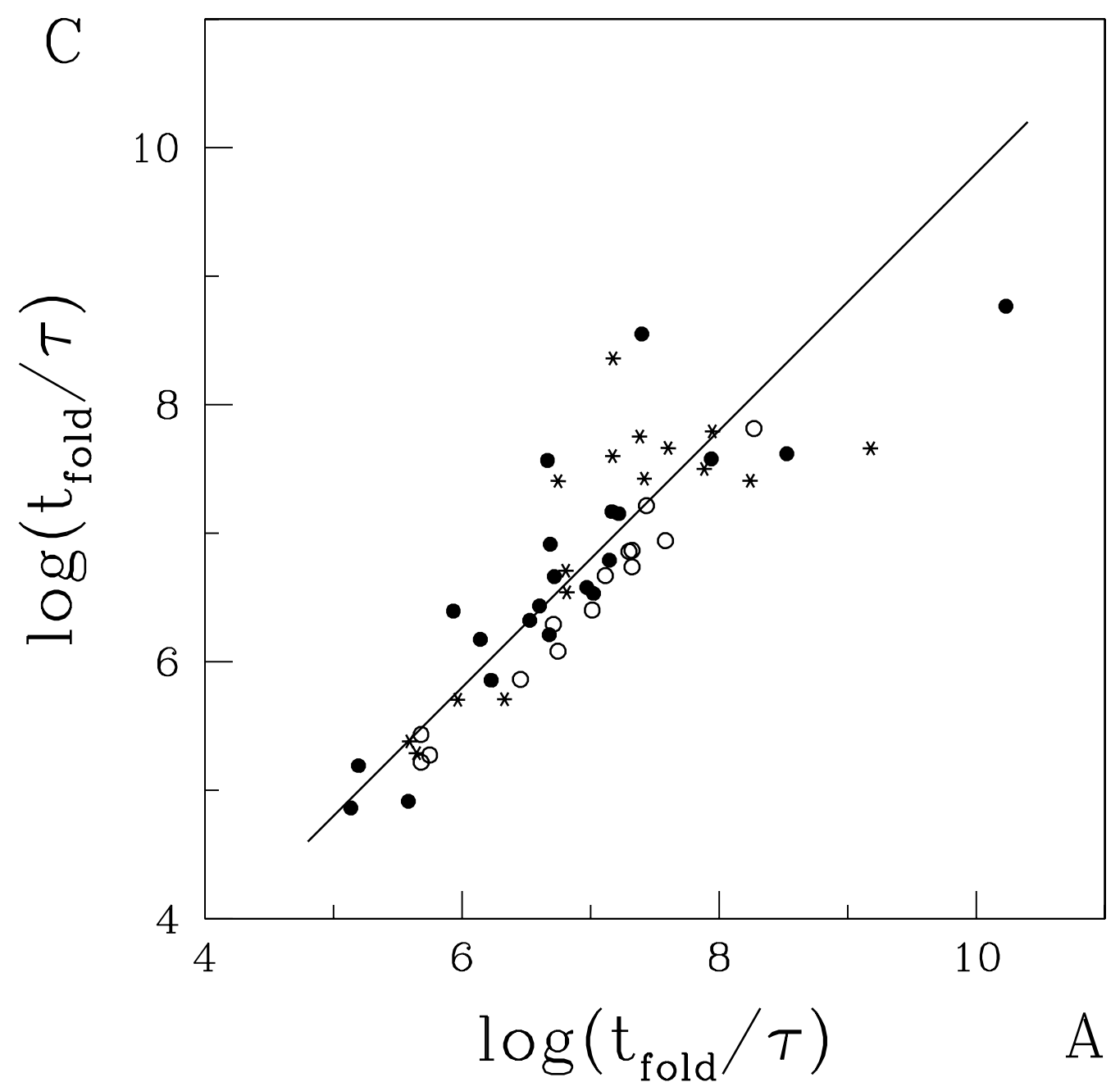

Fig. 2. 


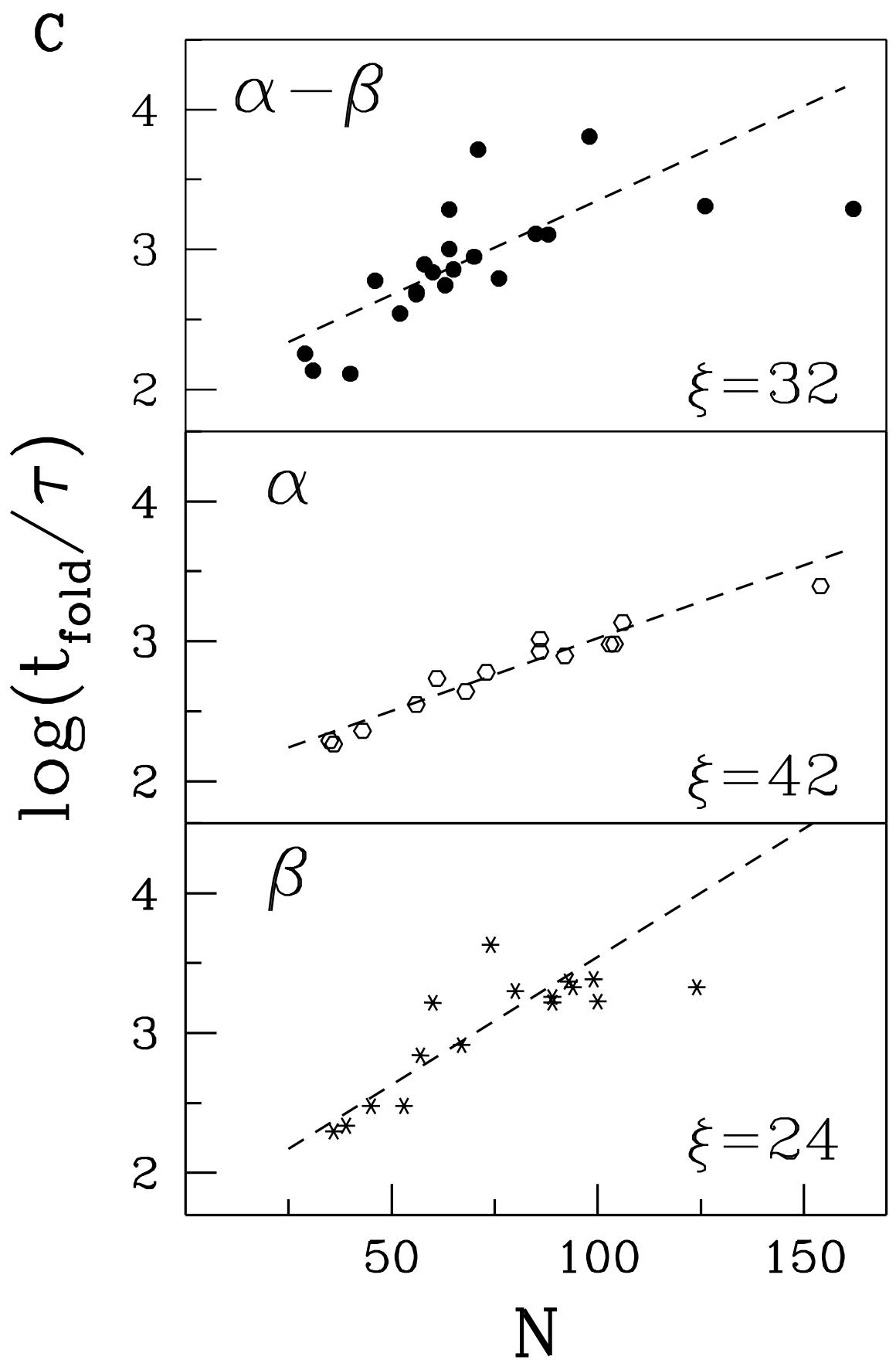

Fig. 3. 

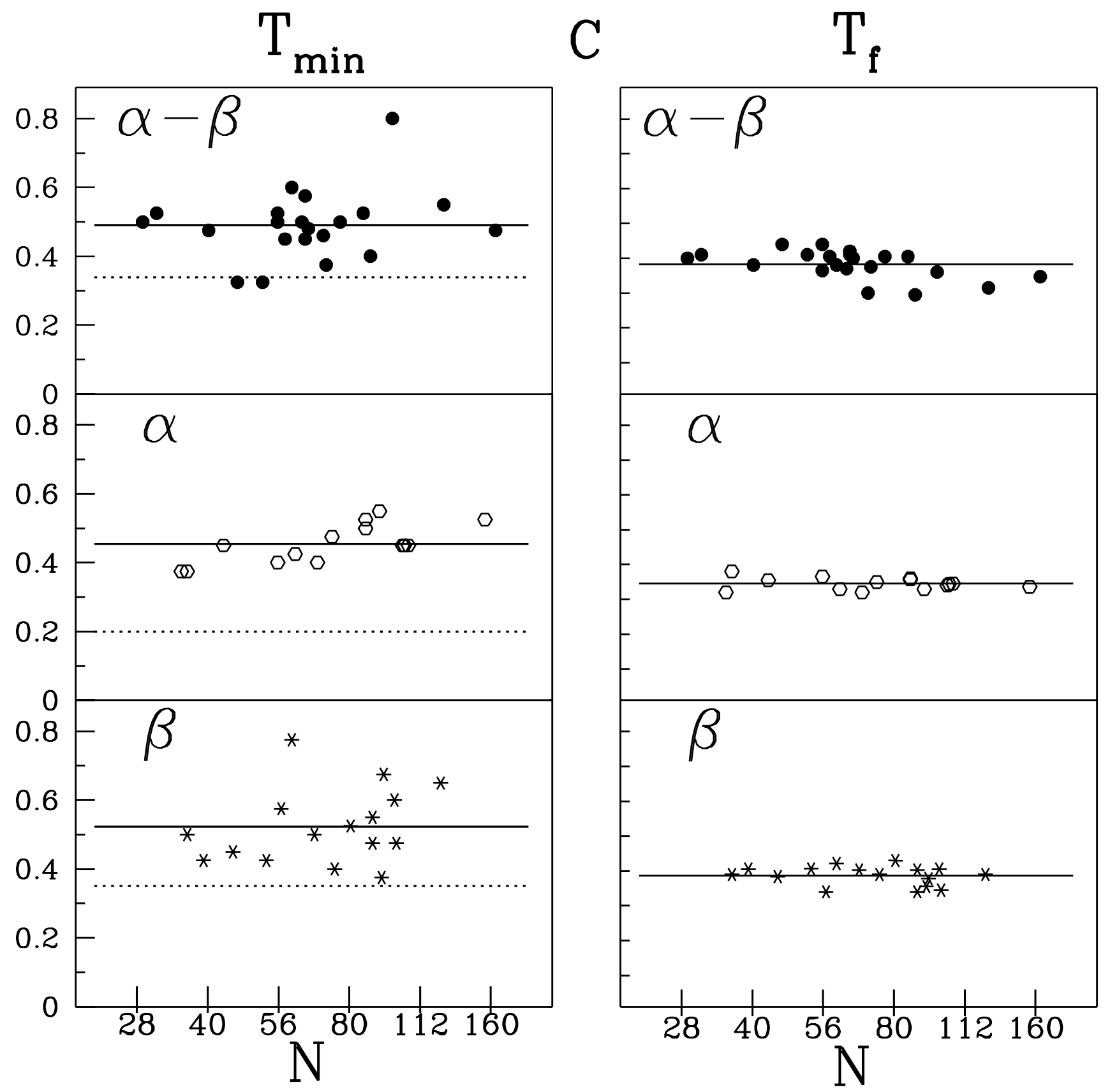

Fig. 4. 


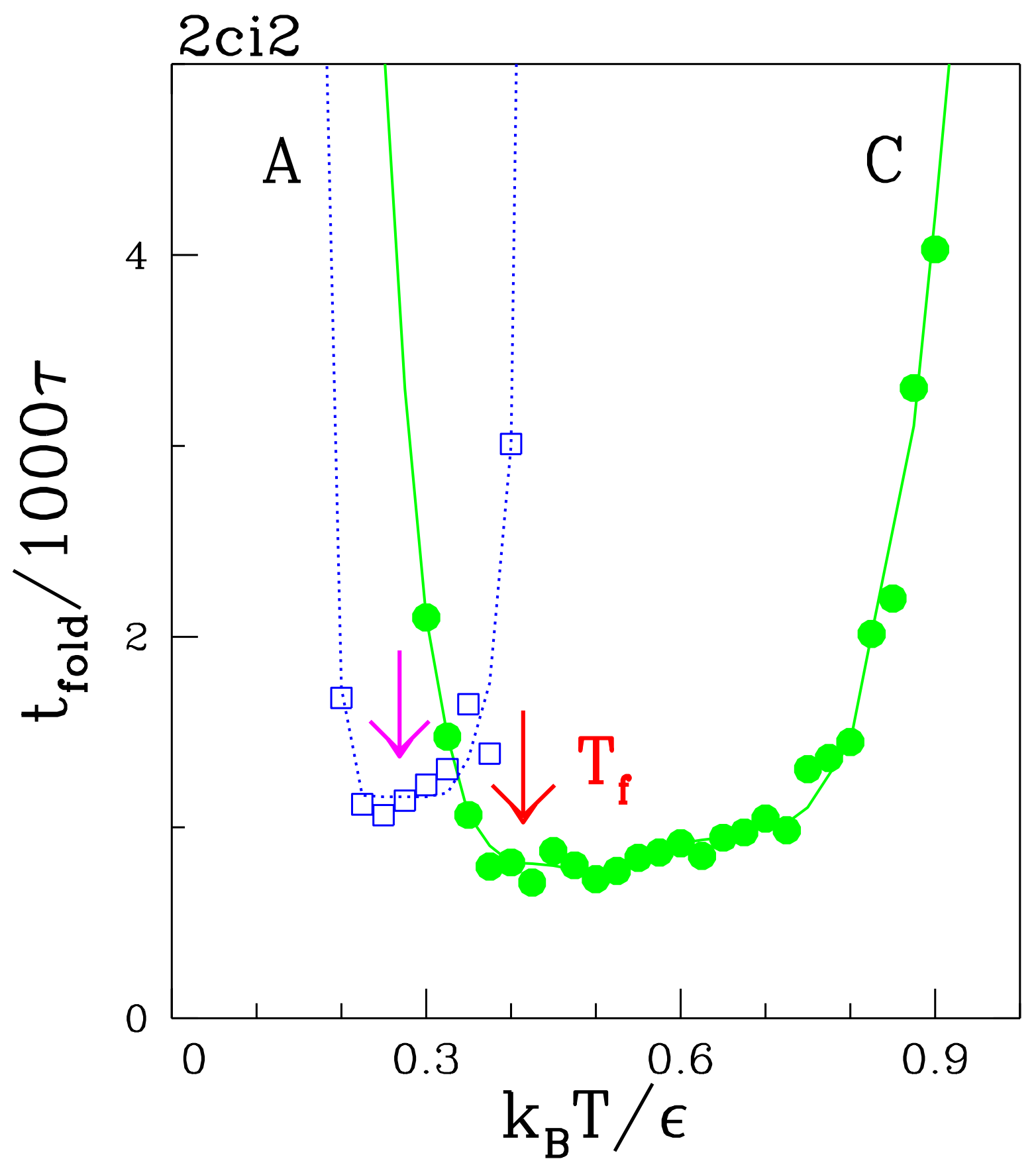

Fig. 5. 


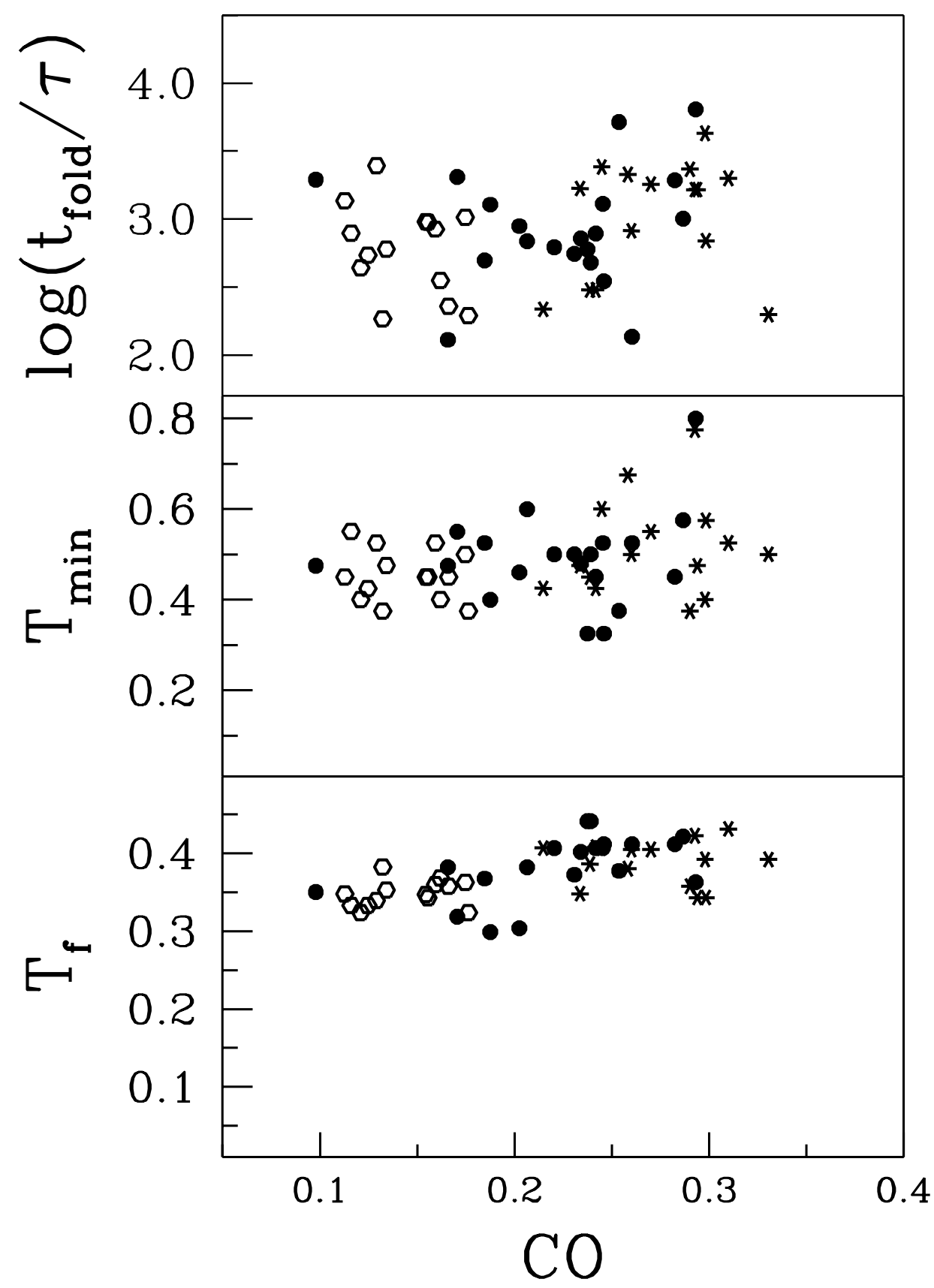

C

Fig. 6 . 


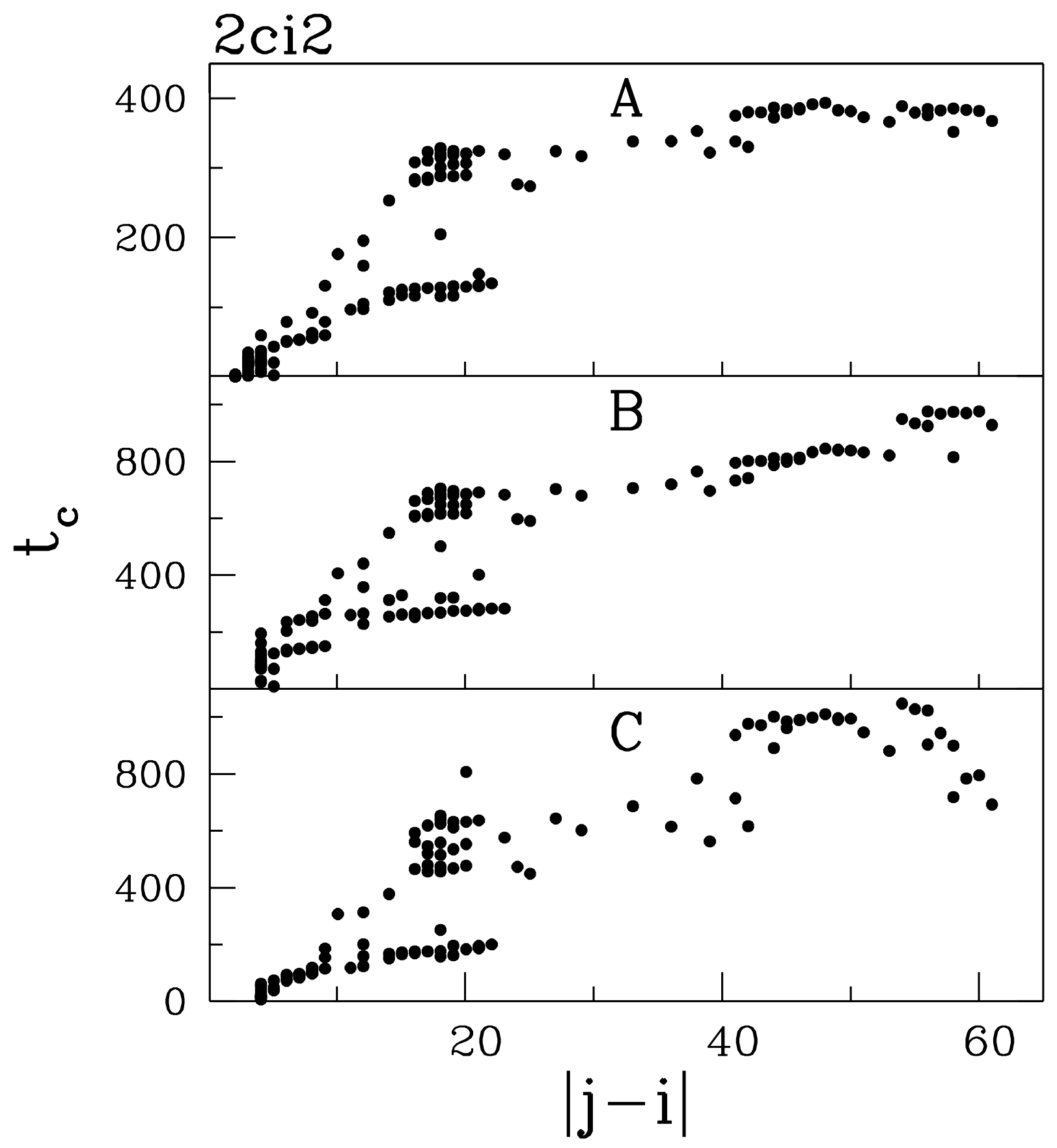

Fig. 7. 

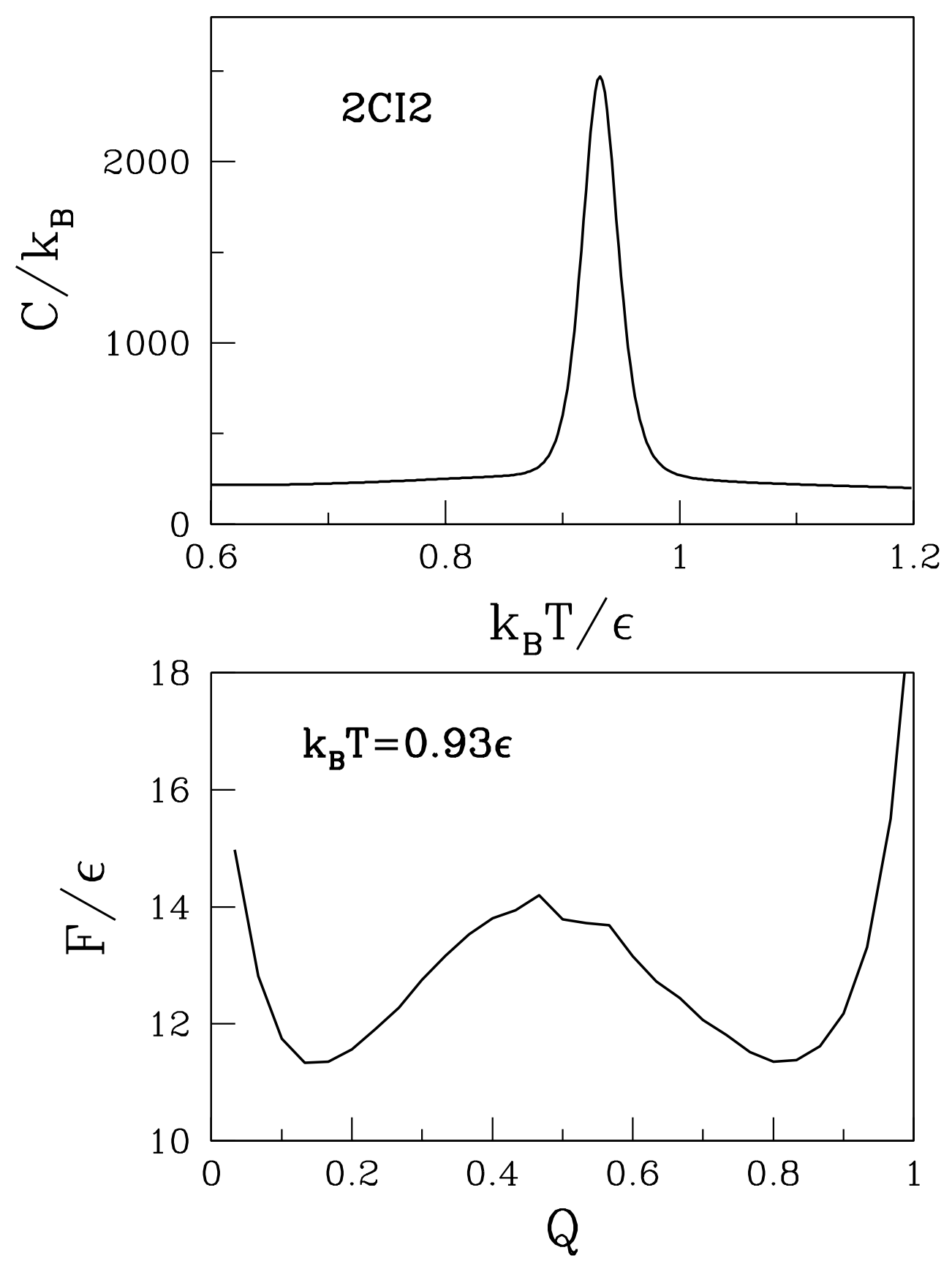

Fig. 8. 


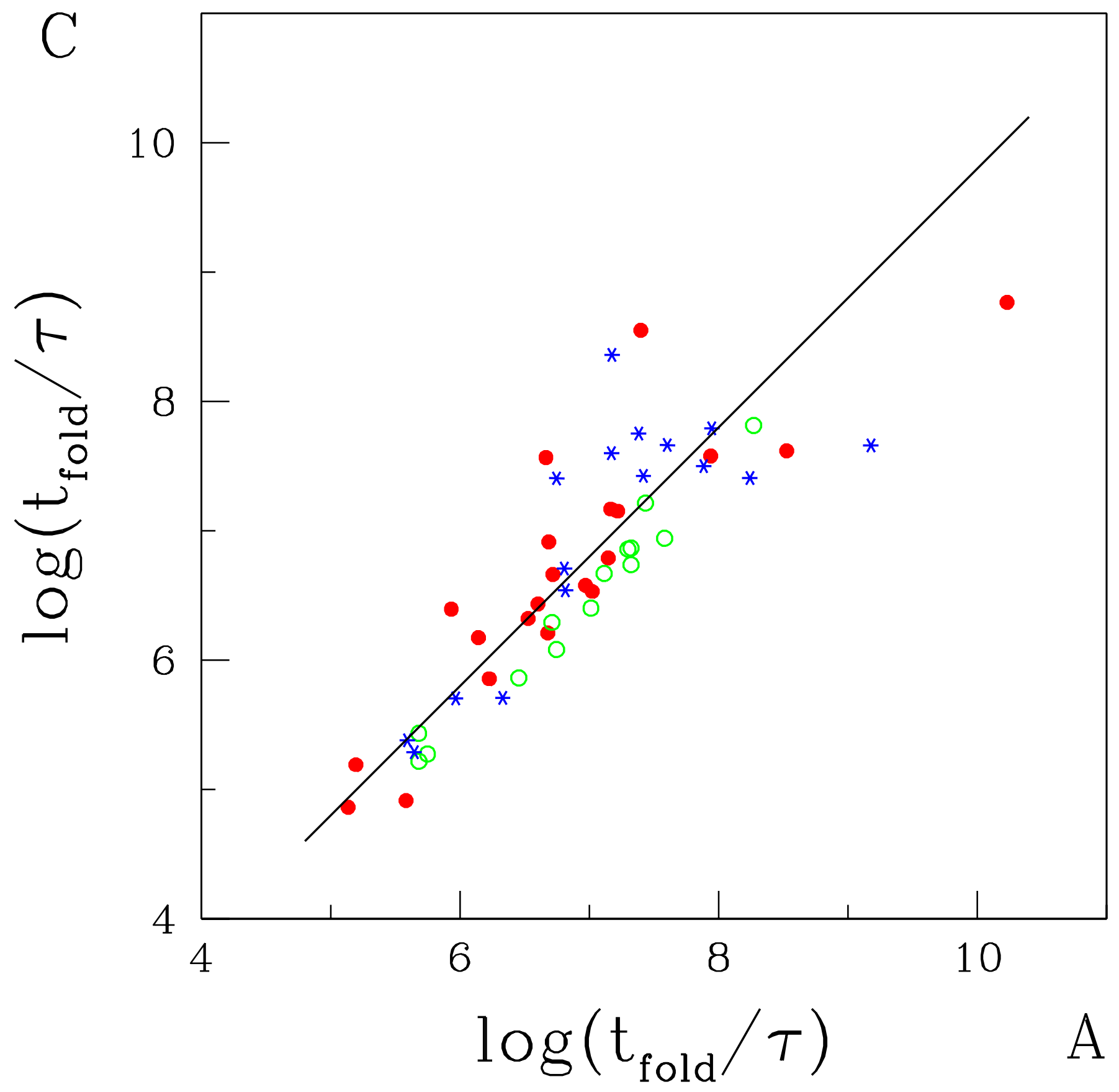




\begin{tabular}{l} 
Abstract \\
Key words: \\
PACS: \\
\hline \\
$\mathbf{1}$ \\
References
\end{tabular}

[1] 